\title{
THE WHOLE TRUTH OF THE MICHELSON-MORLEY EXPERIMENT SLAVIK AVAGYAN
}

\section{Dr. Professor*}

National Polytechnic University of Armenia, GyumriCampus, Armenia E-mail slavikavagyan@yahoo.com

*Corresponding Author: -

E-mailslavikavagyan@yahoo.com

\section{Aristotle}

Abstract: -

The basis of Einstein's relativity theory is the Michelson-Morley experiment. In this paper, based on fundamental laws of physics and mathematics prove the absurdity (inconsistency) of the Michelson-Morley experiment, as it ignores the basic laws of physics.

Keywords: Michelson, Morley, experience, theory, relativity, the law, mathematics, physics, ignoring.

\section{(a) $(\$)$}


The theory of relativity is, in fact, the theoretical proof of Michelson-Morley's experiment. In 1881, Michelson and Morley could not discover the movement of light in the ether. But they could not discover that movement, because that experiment was based on false basis (incorrectly), and besides, light has such property, that in order to discover its movement $10^{-14}$ measurement accuracy should be provided. From this point of view Michelson-Morley's experiment has had a fatal significance as on the basis of the results of this experiment A. Einstein originated his theory of relativity, theory, which is wholly based on incorrect suppositions, judgments and contradictions [ 3 pp.1-3].So, why in the Michelson-Morley experiment the movement of light in the ether has not been detected? On the basis of their experiment the speed at which earth orbits around the sun $(30 \mathrm{~km} / \mathrm{s})$ was considered as movement speed in ether.

The Earth moves in ether at a speed the value and direction of which is practically impossible to determine. In fact, the earth goes not only round the sun at $30 \mathrm{~km} / \mathrm{s}$ speed, but also it goes round the galaxy center with sun at $300 \mathrm{~km} / \mathrm{s}$ speed, the Galaxy in its turn moves at $250 \mathrm{~km} / \mathrm{s}$ speed round a center, and the latter also moves in space at a speed and so on. It means the speed of earth's movement in ether will be the vector sum of these speeds, which is practically impossible to determine. Therefore, Michelson-Morley's experiment is put incorrectly, as it takes into account only one speed, (that of the earth round the Sun) and the most insignificant one. So that the Earth in the air makes a complex motion that almost no one can determine the direction and magnitude of this velocity.

Leaving aside the question of the accuracy of the measurement, we note a major mistake, unsoundness of experience. For clarity, let us bring Feynman's description of the Michelson - Morley experiment of [2 pp.9-12].

"As mentioned above, attempts were made to determine the absolute velocity of the earth through the hypothetical" ether, " that was supposed to pervade all space. The most famous of these experiments is on performed by Michelson and Morley in 1887. It was 18 years later before the negative results of the experiment were finally explained, by Einstein.

The Michelson - Morley experiment was performed with an apparatus like that shown schematically in fig.15-2. This apparatus is essentially comprised of a light source A, a partially silvered glass plate B , and two mirrors C and E, all mounted on a rigid base.

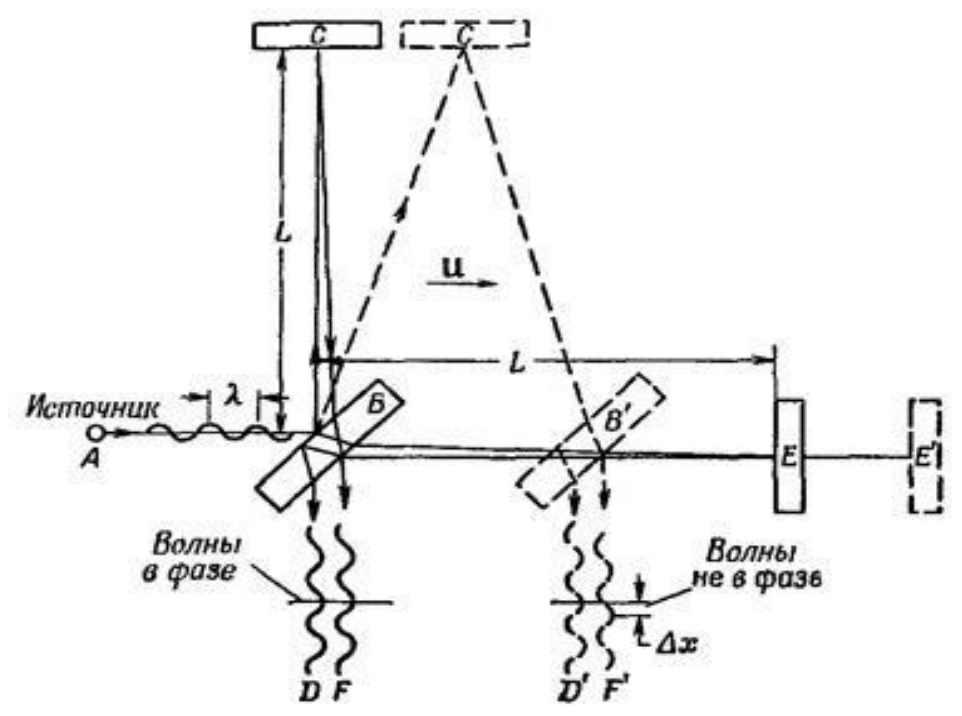

Fig. 15-2 Schematic diagram of the Michelson - Morley experiment

The mirrors are placed at equal distances $\mathrm{L}$ from B. The plate B splits an oncoming beam of light, and two resulting beams continue in mutually perpendicular directions to the mirrors where they are reflected back to B. part of the device: A light, translucent silver-plated glass plate in two mirrors $\mathrm{C}$ and $\mathrm{E}$. All these are rigidly fixed on the heavy plate. Mirrors $\mathrm{C}$ and $\mathrm{E}$ have been placed at the same distance L from the plate B. The plate splits the incident beam of light into two perpendicular to one another; they are directed to the mirror and reflected back to the plate B. Going back through the plate in both the beam overlap ( $\mathrm{D}$ and $\mathrm{F}$ ). If the light transmission time from $\mathrm{B}$ to $\mathrm{E}$ and inversely equal transit time from $\mathrm{B}$ to $\mathrm{C}$ and back, the beams emerging $\mathrm{D}$ and $\mathrm{F}$ will be in phase and mutually intensify; if these times differ even slightly, the beams, the phase shift occurs, and as a consequence - interference. If the device is in the air "at rest", while exactly equal, but if he moves to the right with velocity, there will be a time difference. Let's see why. First, let us calculate the 1 time required for to go from plate $\mathrm{B}$ to mirror $\mathrm{E}$ is $\mathrm{t}_{1}$, and the time for the return is $\mathrm{t}_{2}$.Now, while the lights is on its way from $\mathrm{B}$ to the mirror, the apparatus moves a distanceut ${ }_{1}$, so the light must traverse a distance $\mathrm{L}+\mathrm{ut}_{1}$, at speed $\mathrm{c}$. We can also express this distance as $\mathrm{ct}_{1}$, so we have $\mathrm{ct}_{1}=\mathrm{L}+\mathrm{ut}_{1}$, or $\mathrm{t}_{1}=\mathrm{L} /(\mathrm{c}-\mathrm{u})$. (This result is also obvious from the point of view that velocity of light relative to the apparatus is $\mathrm{c}-\mathrm{u}$, so the time is the length $\mathrm{L}$ divided by $\mathrm{c}-\mathrm{u}$ ). In a like manner, the team $\mathrm{t}_{2}$ can be calculated. During this time the plate B advances a distance $\mathrm{ut}_{2}$, so the return distance of the light is L-ut 2 . Then we have

$\mathrm{ct}_{2}=\mathrm{L}-\mathrm{ut}_{2}$, or $\mathrm{t}_{2}=\mathrm{L} /(\mathrm{c}+\mathrm{u})$.

The total time is

$$
t_{1}+t_{2}=\frac{2 L c}{c^{2}-u^{2}}
$$


For convenience in later comparison of times we write this as

$$
t_{1}+t_{2}=\frac{2 L / c}{1-u^{2} / c^{2}} .
$$

Our second calculation will be the time $\mathrm{t}_{3}$ for the light to go from $\mathrm{B}$ to the mirror $\mathrm{C}$. As before, during time $\mathrm{t}_{3}$ the mirror $\mathrm{C}$ moves to the right a distance ut 3 to the position $\mathrm{C}^{\prime}$; in the same time, the light travels a distance $\mathrm{ct}_{3}$ along the hypotenuse of triangle, which is $\mathrm{BC}^{\prime}$. For this right triangle we have

$\left(\mathrm{ct}_{3}\right)^{2}=\mathrm{L}^{2}+\left(\mathrm{ut}_{3}\right)^{2}$, or

$\mathrm{L} 2=\mathrm{c} 2 \mathrm{t} 32-\mathrm{u} 2 \mathrm{t} 32=(\mathrm{c} 2-\mathrm{u} 2) \mathrm{t} 32$, from which

we get

$$
\mathrm{t}_{3}=\frac{\mathrm{L}}{\sqrt{\mathrm{c}^{2}-\mathrm{u}^{2}}}
$$

For the return trip from $C^{\prime}$ the distance is the same, as can be seen from the symmetry of the figure; therefore the return time is also the same, and, and the total time is $2 \mathrm{t}_{3}$. With a little rearrangement of the form we can write

$$
2 t_{3}=\frac{2 L}{\sqrt{c^{2}-u^{2}}}=\frac{2 L / c}{\sqrt{1-u^{2} / c^{2}}} \text {. }
$$

In carrying out the experiment, Michelson and Morley oriented the apparatus so that the line BE was nearly parallel to the Earth's motion in its orbit, but (at certain times of the day and night). This orbital speed is about $30 \mathrm{~km} / \mathrm{s}$, and any " ether drift " should be at least that much at some time during the year. The apparatus was amply sensitive to observe such an effect, but no time difference was found - the velocity of the Earth through the ether could not be detected. The result of the experiment was null.

The result of the Michelson-Morley experiment was very puzzling most disturbing. The first fruitful idea how finding a way out of the impasse came from Lorenz. . .

So if the apparatus shrinks in the manner just described, we have a way of understanding why the Michelson-Morley experiment gives no effect at all" ...

But the "mystery" is that in the experiment Michelson-Morley ignored the laws of optics, namely, the law of reflection of light. This law is also present in the book [2, pp .246]

_... (Fig. 26.1). The simplest object is a mirror, and the law for a mirror is that when the light hits the mirror, it does not continue in a straight line, but bounces off the mirror into a new straight line, which changes when we changes the inclination of the mirror. The question for the ancients was, what is the relation between the two angles involved?

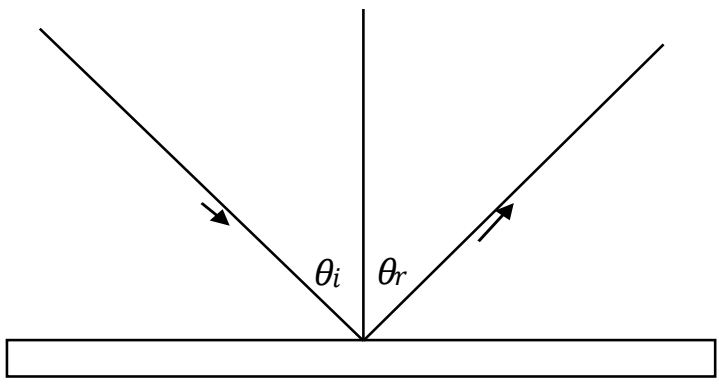

Fig.26.1. The angle of incidence equals the angle of reflection.

This is a very simple relation, discovered long ago. The light striking a mirror travels in such a way that the two angles , between each beam and the mirror, are equal. For some reasons it is customary to measure the angles from the normal to the mirror surface. Thus the so-called law of reflection is

$$
\theta \mathrm{i}=\theta \mathrm{r} » .
$$

But in the Michelson-Morley experiment, this fundamental law is neglected.

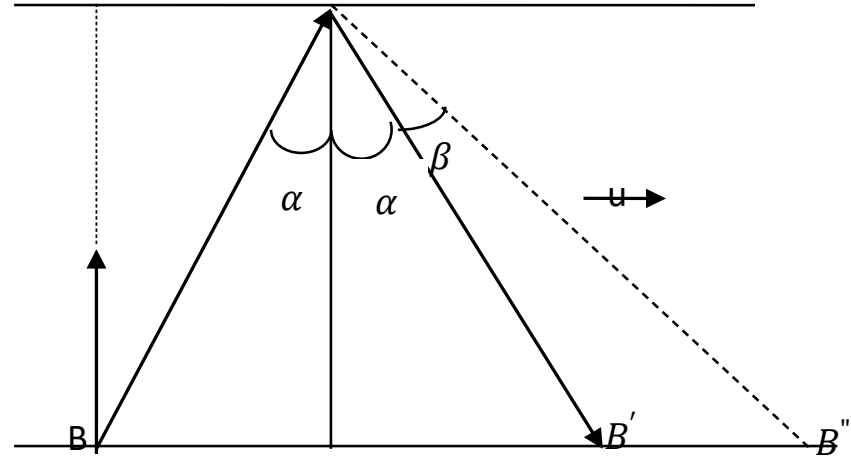

Fig. 1 
And so what happens: In the Michelson-Morley experiment from point $\mathrm{B}$ to $\mathrm{C}$ beam is directed perpendicular to the motion of the system. As a result of the addition of velocities $\boldsymbol{c}$ cand $\boldsymbol{u}$ light beam deviates from the vertical and goes on the direction of $\mathrm{BC}$, which forms a vertical angle $\alpha$. So the beam falls on the mirror $\mathrm{C}$ at an angle $\alpha$. What happens next at the point $\mathrm{C}$ ? According to the law of reflection of light from point $\mathrm{C}$ will go a beam of light at an angle $\alpha$ as well.

That is, from point $\mathrm{C}$ light beam already goes out not vertically. That's the whole mistake.

Then as a result of addition of velocities $\boldsymbol{C}$ and $\boldsymbol{u}$, the light beam (figure 1) from the point $\mathrm{C}$ goes not in the direction $\mathrm{CB}^{\prime}$, but in some other direction $\mathrm{CB}^{\prime \prime}$, which with $\mathrm{CB}^{\prime}$ forms an angle $\beta$. By the way, angle $\beta$ can be determined with simple calculations:

The beam reflected from the point $\mathrm{C}$, if the system has been fixed, travels a distance $\mathrm{C} B^{\prime}=c t$ during time $\mathrm{t}$,

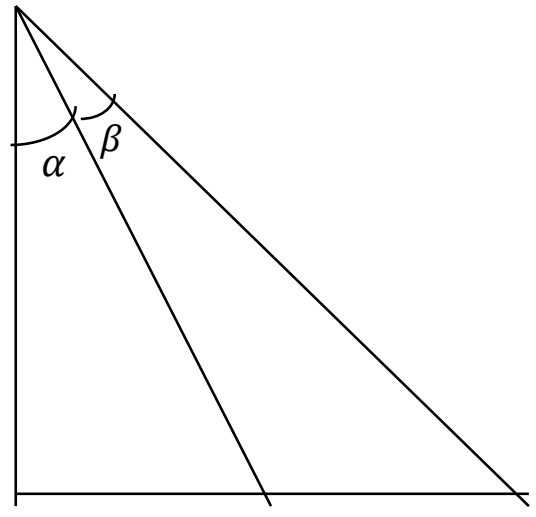

Then $\mathrm{OB}^{\prime}=$ ct $\sin \alpha$.

Since the system carries light along with the velocity $\mathrm{u}, \operatorname{then} B^{\prime} B^{\prime \prime}=u t$.

From the triangle $\mathrm{OCB}^{\prime}$ we have $\mathrm{OC}=\mathrm{CB}^{\prime} \cos \alpha=\mathrm{ct} \cos \alpha .(1)$

From the triangle $\mathrm{OCB}^{\prime \prime}$ we havetan $(\alpha+\beta)={ }^{\mathrm{OB}}{ }^{\prime}$, where OC

$$
\mathrm{OC}=\frac{\mathrm{ctsin} \alpha+\mathrm{ut}}{\tan (\alpha+\beta)}
$$

Solving (1) and (2), we obtain

$$
\tan \beta=\frac{u}{c \cos \alpha+c \cos \alpha(\tan \alpha)^{2}+u \tan \alpha}
$$

Since $\mathrm{BC} \neq \mathrm{CB} "$, the distance which the light will pass from point $\mathrm{B}$ to the mirror $\mathrm{C}(\mathrm{BC})$, is not equal to the distance which the reflected light will pass from the mirror $\mathrm{C}$ to the point $\mathrm{B}(\mathrm{CB} ")$.

Since $\mathrm{BC} \neq \mathrm{CB}$ ", it is clear that it is impossible to deduct the time dilation equation necessary for Einstein's theory:

$$
t=\frac{t_{0}}{\sqrt{1-\frac{v^{2}}{c^{2}}}}
$$

The same applies to the equation of the length contraction. Thus two basic ideas that underlie relativity theory, time dilation and length contraction, are groundless.

This is the fundamental fallacy of the Michelson-Morley experiment. The laws of mathematics and physics are the basis of science. To disregard the arguments proved by the same laws, to put it mildly unethical. To neglect these powerful tools that are at the basis of all science, just brings great damage to science. 


\section{References}

[1] V. V. Sobolev, "Course of Theoretical Astrophysics," Moscow, 1967

[2] Feynman, R. Leyton, M. Sends The Feynman Lectures on Physics. vol.1 Mainly mechanics, radiation, and heat. Addison-Wesley publishing company. 1963

[3] Slavik Avagyan "On the experiment of Michelson-Morley" 4th International Scientific Conference on Physics and Control. PhysCon 2009. Catania, Italy, September 1-4 2009. lib.physcon.ru/file?id=c18891e8c27a 